\title{
Effect of Neuromuscular Electrical Stimulation on Gluteus Maximus and Quadriceps in Cerebral Palsy Children with Crouch Gait
}

\section{Patitapaban Mohanty ${ }^{*}$, Monalisa Pattnaik and Anjushree Sarkar}

Swami Vivekanand National Institute of Rehabilitation Training and Research, Olatpur, Bairoi, Cuttack, India

*Corresponding author: Patitapaban Mohanty, Swami Vivekanand National Institute of Rehabilitation Training and Research, Olatpur, Bairoi, Cuttack, India, Tel: +91 9437487139; E-mail: ppmphysio@rediffmail.com

Rec date: Jun 08, 2016; Acc date: Jun 28, 2016; Pub date: Jun 30, 2016

Copyright:@ 2016 Mohanty P, et al. This is an open-access article distributed under the terms of the Creative Commons Attribution License, which permits unrestricted use, distribution, and reproduction in any medium, provided the original author and source are credited.

\begin{abstract}
Background: Cerebral palsy (CP) is a persistent disorder of movement and posture caused by non-progressive pathological processes of the immature brain. This is the natural history of the gait disorder in children with more severe diplegia and in the majority of children with spastic quadriplegia. One of the most common movement abnormalities among children with cerebral palsy is a crouched gait. Neuromuscular electrical stimulation (NMES) is the application of electrical current transcutaneously to innervated, superficial muscle to stimulate muscle fibers, augment muscle contraction, increase range of motion (ROM), and increase sensory awareness. The purpose of the study was to investigate the effects of NMES on Gluteus maximus and Quadriceps strength in cerebral palsy children with crouch gait, and the subsequent effect on their gross motor function.
\end{abstract}

Methods: Total 40 children (13 females, 27 males, age group 5 years to 11 years) were recruited for study from the paediatric section of physiotherapy department of Swami Vivekanand National Institute of Rehabilitation Training and Research according to the inclusion and exclusion criteria and were randomly allotted in 2 groups. All were randomly assigned to either the stimulation (group 1) or control group (group 2). The stimulation group received neuromuscular electrical stimulation to gluteus maximus and quadriceps muscles for 15 minutes each, 5 days/week for a period of 6 weeks.

Results: Both the groups showed significant improvement in strength of gluteus maximus and quadriceps of CP children with crouch gait, but experimental group showed significantly more improvement as compared to control group. There was also statistically significant difference in dimension $D$ and $E$ (standing and walking) of the gross motor function measure between the experimental group and control group.

Conclusion: This study has shown that neuromuscular electrical stimulation in addition to conventional exercise is found to be effective in improving strength and function than conventional exercise alone.

Keywords: Cerebral palsy; Crouch gait; Neurological surgery

\section{Introduction}

Cerebral palsy $(\mathrm{CP})$ is a persistent disorder of movement and posture caused by non-progressive pathological processes of the immature brain. The prevalence of $\mathrm{CP}$ is between 1.5 and 2.5 per 1000 live births and spastic subtypes are represented in $64.6 \%$ of patients with CP [1].

This is the natural history of the gait disorder in children with more severe diplegia and in the majority of children with spastic quadriplegia. One of the most common movement abnormalities among children with cerebral palsy is a crouched gait [2].

Crouch gait, a common movement pattern in individuals with cerebral palsy, is characterized by excessive dorsiflexion or calcaneus at the ankle in combine with excessive flexion at the knee and hip [2]. It suggests the failure of the mechanisms responsible to maintain the body in an upright position. The three muscle groups known as the antigravity muscles are the hip extensors (gluteus maximus and hamstrings), the knee extensors (vasti) and the ankle plantar flexors (soleus). These muscles are primarily responsible for keeping the body in the upright position. Inability of one or more of these muscles to function adequately results in a crouch gait and is observed as body collapsing into a flexion posture [3].

Lever arm dysfunction also contributed to crouch gait. With hip and knee in flexed position, as seen in crouch, the lever arm at knee becomes longer compared to that at the hip. Hence, hamstrings now function more effectively as knee flexors than as hip extensors. With increasing knee flexion, lever arm of hamstrings as knee flexor increases and with increasing hip flexion lever arm for rectus as hip flexor increases.

Neuromuscular electrical stimulation (NMES) is the application of electrical current transcutaneously to innervated, superficial muscle to stimulate muscle fibers, augment muscle contraction, increase range of motion (ROM), and increase sensory awareness [4-6]. Electrical stimulation as a treatment option for cerebral palsy $(\mathrm{CP})$ has been proposed after several studies in recent years.

Strength training programs designed for individuals with cerebral palsy and crouch gait have targeted the hip and knee extensors [7]. It improves strength in targeted muscles without increased spasticity $[8,9]$ and has positive effect on gait and gross motor function [7]. 
Therefore, the present study intends to evaluate the effect of neuromuscular electrical stimulation on gluteus maximus and quadriceps in cerebral palsy children with crouch gait by conducting pre-test and post-test evaluations of the treatment effects.

\section{Methodology}

Total 40 children were recruited for study from the paediatric section of physiotherapy department of Swami Vivekanand National Institute of Rehabilitation Training and Research according to the inclusion and exclusion criteria and were randomly allotted in 2 groups. To be included in the study, the child had to meet the following inclusion criteria: Children diagnosed as spastic cerebral palsy, age group 5 years to 11 years, able to walk in crouch gait with or without support, cognitive function is normal or near normal, able to accept and follow verbal instructions and cooperative. Exclusion criteria were children with mental retardation, children with spastic hemiplegia, athetoid, ataxic cerebral palsy, child with instable seizures and behavioral disorders, musculoskeletal or neurological surgery within 1 year prior to study, botulinum toxin injections within 6 months prior to study.

\section{Procedure}

Spastic children with crouch gait with or without support were evaluated. After meeting the inclusion and exclusion criteria, informed consent was taken from the parents/caregivers of the subjects and then children were randomly allocated to either of the two groups. Group 1: neuromuscular stimulation and conventional exercise (experimental group) ( $n=20)$, Group 2: conventional exercise (control group) $(n=20)$ were treated by conventional exercises.

Children were given stimulation in side lying position for gluteus maximus and in sitting position for quadriceps. During the application of neuromuscular electrical stimulation, the child was positioned with the knee flexed (70-80 degree) and the hamstring was not in the lengthened position to reduce the amount of stimulation required to attain a forceful contraction and therefore improve comfort. The intervention period was of 6 weeks duration, neuromuscular stimulation for 15 minutes each muscles, 5 days/week. Parameters used: Waveform- balance, symmetrical, biphasic; Pulse duration - 300 ms; Frequency - 50 pulse/sec; Stimulation time: Rest time - on: off - 5: 15; Ramp up - $1 \mathrm{sec}$, Ramp down-1 sec; Intensity - maximum tolerable intensity was used to produce a visible contraction but as tolerated by the child.

\section{Data collection}

Gluteus maximus and Quadriceps muscle strength was measured by modified sphygmomanometer and gross motor function measure were measured by GMFM (dimension D and E). The data was collected blindly by two testers independently throughout the study. Pre-test measurement was taken on recruitment of the subject and Post-test measurement was taken after 6 weeks of treatment.

\section{Data analysis}

Data were analyzed using $2 \times 2$ ANOVA with one between factor (group) with two levels (pre-test and post-test) and one within factor (time) with two levels (pre-test and post-test) for strength. Between groups difference for GMFM was done by Mann Whitney U test. An alpha level of 0.05 of significance was set. Analysis was performed using SPSS package 16 version.

\section{Results}

The combined effect of electrical stimulation with conventional therapy shows improvement in strength of gluteus maximus and quadriceps in cerebral palsy children with crouch gait, this also shows the overall improvement as a measure of gross motor function in GMFM. Strength of gluteus maximus and quadriceps muscles in both the groups improved significantly however at the end of the treatment experimental group showed significantly more improvement. The subjects in the experimental group showed more significant change than control group in their gross motor function.

\section{Strength of Gluteus maximus (Left)}

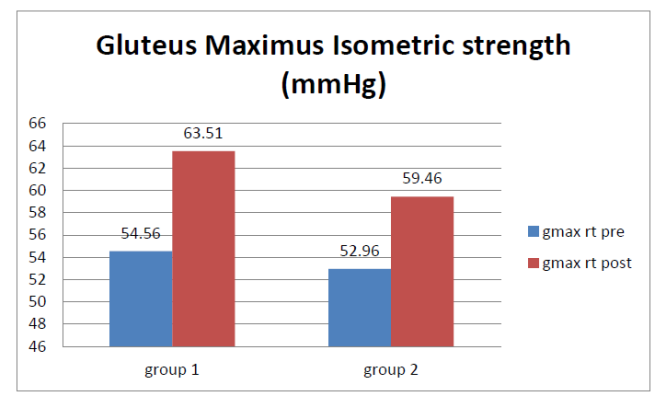

Figure 1: Change in strength of gluteus maximus. gmax rt pregluteus maximus right pre, gmax rt post-gluteus maximus right post.

Figure 1 illustrates that both the group's experimental (NMES + Conventional therapy) and control (Conventional therapy) improved in strength from pre to post treatment measurements after 6 weeks.

There was main effect for time, $\mathrm{f}(1,38 ; 0.05)=347.893, \mathrm{p}<0.001$ and a main effect for time $\mathrm{x}$ group interaction, $\mathrm{f}(1,38 ; 0.05)=11.396$, $\mathrm{p}<0.002$.

There was no main effect for group as $\mathrm{f}(1,38 ; 0.05)=0.168, \mathrm{p}<0.684$.

Post Hoc analysis showed that both the groups improved significantly however at the end of the treatment experimental group showed significantly more improvement.

\section{Strength of Gluteus maximus (Right)}

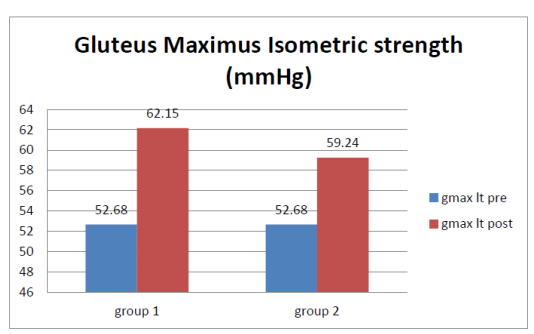

Figure 2: Change in strength of gluteus maximus. gmax It pregluteus maximus left pre, gmax It post- gluteus maximus left post. 
Figure 2 illustrates that both the group's experimental (NMES + Conventional therapy) and control (Conventional therapy) improved in strength from pre to post treatment measurements after 6 weeks.

There was main effect for time, $f(1,38 ; 0.05)=248.560, p<0.001$ and a main effect for time $\times$ group interaction, $\mathrm{f}(1,38 ; 0.05)=6.242$, $\mathrm{p}<0.017$.

There was no main effect for group as $\mathrm{f}(1,38 ; 0.05)=0.615$, $\mathrm{p}<0.438$.

Post Hoc analysis showed that both the groups improved significantly however at the end of the treatment experimental group showed significantly more improvement.

\section{Strength of Quadriceps (Left)}

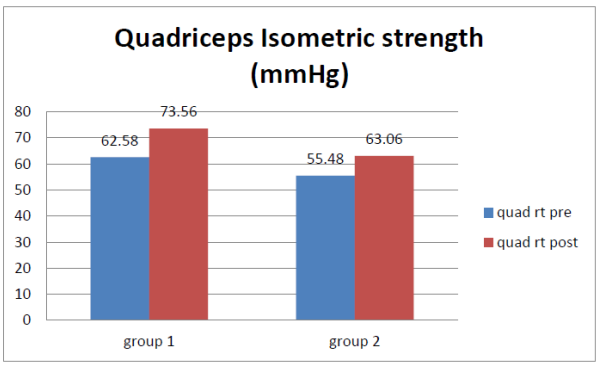

Figure 3: Change in strength of quadriceps. Quad rt pre-quadriceps right pre, quad rt post-quadriceps right post

Figure 3 illustrates that both the group's experimental (NMES + Conventional therapy) and control (Conventional therapy) improved in strength from pre to post treatment measurements after 6 weeks.

There was main effect for time, $\mathrm{f}(1,38 ; 0.05)=281.721, \mathrm{p}<0.001$ and a main effect for time $\mathrm{x}$ group interaction, $\mathrm{f}(1,38 ; 0.05)=11.347$, $\mathrm{p}<0.002$.

There was no main effect for group as $\mathrm{f}(1,38 ; 0.05)=2.696, \mathrm{p}<0.109$.

Post Hoc analysis showed that both the groups improved significantly however at the end of the treatment experimental group showed significantly more improvement.

\section{Strength of Quadriceps (Right)}

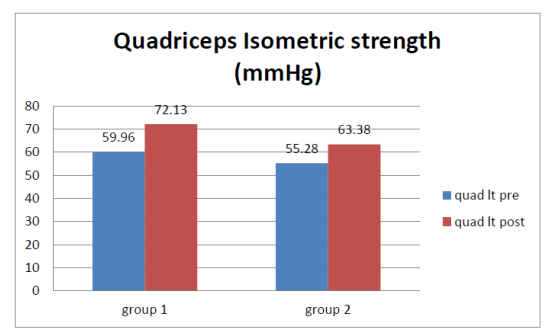

Figure 4: Change in strength of quadriceps. Quad lt pre- quadriceps left pre, quad lt post- quadriceps left post.

Figure 4 illustrates that both the group's experimental (NMES + Conventional therapy) and control (Conventional therapy) improved in strength from pre to post treatment measurement after 6 weeks.
There was main effect for time, $\mathrm{f}(1,38 ; 0.05)=240.193, \mathrm{p}<0.001$ and a main effect for time $\mathrm{x}$ group interaction, $\mathrm{f}(1,38 ; 0.05)=8.052$, $\mathrm{p}<0.007$. Also there was main effect for group as $\mathrm{f}(1,38 ; 0.05)=4.153$, $\mathrm{p}<0.049$.

Post Hoc analysis showed that both the groups improved significantly however at the end of the treatment experimental group showed significantly more improvement.

\section{Gross Motor Function Measure (GMFM)}

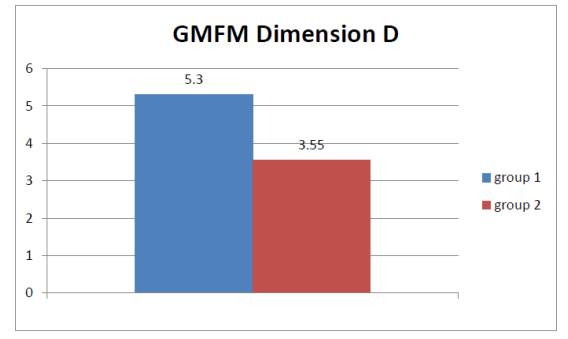

Figure 5: Change in GMFM-D scores.

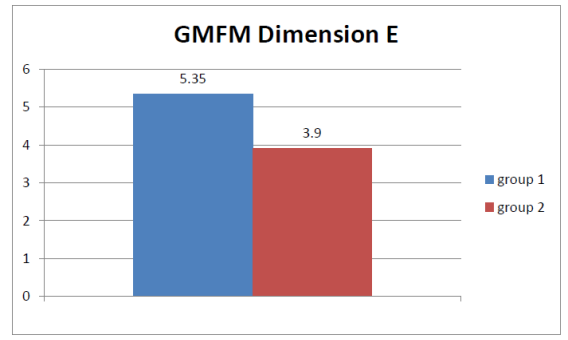

Figure 6: Change in GMFM-E scores.

Figures 5 and 6 demonstrate the subjects in the experimental group showed more significant change than control group in their gross motor function. There is statistically significant difference in dimension D (standing) of the gross motor function measure in the experimental group with the significance level of 0.003, Mann Whitney $\mathrm{U}$ score: 91.5 and $\mathrm{Z}$ score: -3.007 and dimension $\mathrm{E}$ (walking) of the gross motor function measure in the experimental group with significance level of 0.011 , Mann Whitney U score: 109.5 and Z score: -2.550 .

\section{Discussion}

Overall results of the study show both groups improved significantly for strength of gluteus maximus and quadriceps (left and right side). However, the improvement was significantly more in experimental group. The change in function, GMFM Dimension D and E, from pre to post was also significantly more in experimental group.

Both the groups improved in 6 weeks treatment for strength but the improvement was more in stimulation group i.e., In group 1 gluteus maximus strength increased by $17.98 \%$ (left) and $16.4 \%$ (right), quadriceps strength increased by $20.3 \%$ (left) and $17.55 \%$ (right). In group 2 gluteus maximus strength increased by $12.45 \%$ (left) and $12.27 \%$ (right), quadriceps strength increased by $14.65 \%$ (left) and $13.66 \%$ (right). 
Gross motor function measure (GMFM)- The present study shows that there was significant improvement in function in both the groups but subjects in the experimental groups showed more significant change than control group in their gross motor function, dimension $\mathrm{D}$ with mean ranks 5.3 and 3.5 and dimension $\mathrm{E}$ with mean ranks 5.35 and 3.9 respectively.

The experimental group showed improvement greater than control group; the reason behind it, children with $\mathrm{CP}$ are unable to fully activate their voluntary muscles as compared to typically developing children, NMES, an alternative strength training technique, it actually excites the motor nerve going to muscle and causing the muscles to contract with Maximum voluntary isometric contraction (MVIC) force and increase cross-sectional area (CSA) of the muscle. Strength training by NMES does promote neural and muscular adaptations. Strength and function are directly proportional to each other as evident by previous studies.

The result of the present study is found to be similar to the study done by ML van der Linden [10] on Electrical stimulation of Gluteus maximus in children with cerebral palsy: effects on gait characteristics and muscle strength to determine whether electrical stimulation of the gluteus maximus would improve hip extensor strength in children with cerebral palsy (CP). Twenty-two ambulant children (diplegic, hemiplegic, and quadriplegic) were randomly assigned to either the stimulation or control group. The stimulation group received electrical stimulation of the gluteus maximus of the most affected legs for 1 hour a day, 6 days a week for a period of 8 weeks. Measurements of hip extensors strength were made before and after treatment for both groups. And found that both groups were improved equally in strength. Daichman et al. [11] did a study on the effect of NMES home program on impairments and functional skills of a child with spastic diplegic $\mathrm{CP}$ to examine the effects of neuromuscular electrical stimulation (NMES) on impairments and functional skills of a 13-yearold child with spastic diplegic cerebral palsy (CP). NMES was administered to the right quadriceps muscles every other day for six weeks. Pre and post testing included assessment of strength using a hand-held dynamometer. After intervention, right quadriceps strength increased from $16.3 \pm 3.06$ to $41.7 \pm 4.2$ Newtons. A study was done by Karen J. Dodd et al. [7] on a systemic review of the effectiveness of strength training programs for people with cerebral palsy to determine whether strength training is beneficial for people with cerebral palsy. The articles are selected on the basis of (i) population (people with $\mathrm{CP}$ ), (ii) intervention (strength training or a progressive resistance exercise program), and (iii) outcomes (changes in strength, activity, or participation) and found that training can increase strength and may improve motor activity in people with cerebral palsy without any adverse effect. Dr. Lalit Arora et al. [12] studied effect of electrical stimulation on spasticity in spastic diplegic cerebral palsy children and results showed that decreased spasticity and improved knee ROM. Camrick J. [5] studied clinical use of neuromuscular electrical stimulation for children with cerebral palsy and results showed that the functional changes that occur with the application of neuromuscular electrical stimulation to the lower extremity may be a useful physical therapy tool when used with task-oriented functional activities.
Electrical stimulation may enhance muscle contraction and provide sensation so that a child can add a weak response with effective results and assist in improving motor control.

\section{Conclusion}

The study revealed that the effect of conventional strengthening exercise protocol along with Neuromuscular Electrical Stimulation (NMES) in cerebral palsy children with crouch gait is more effective than conventional strengthening exercise protocol alone to improve muscle strength and motor function.

\section{Limitation}

Small sample size, Carry over effect of the study has not been studied.

\section{References}

1. Kamper DG, Yasukawa AM, Barrett KM, Gaebler-Spira DJ (2006) Electrostimulation at sensory level improves function of the upper extremities in children with cerebral palsy: a pilot study. Pediatr Phys Ther 18: 31-38

2. Rodda J, Graham HK (2001) Classification of gait patterns in spastic hemiplegia and spastic diplegia: A basis for a management algorithm. Eur J Neurol 8 Suppl 5: 98-108.

3. Ganjwala D (2009) Orthopaedic Hospital, Crouch Gait in Cerebral Palsy; Official newsletter of the Paediatric Orthopaedic Society of India.

4. Baker LL, McNeal DR, Benton LA, (2000) Neuromuscular electrical stimulation. A Practical Guide. (4thedn). Downey, CA: Los Amigos Research and Education Institute.

5. Carmick J (1993) Clinical use of neuromuscular electrical stimulation for children with cerebral palsy, Part 1: Lower extremity. Phys Ther 73: 505-513.

6. Carmick J (1993) Clinical use of neuromuscular electrical stimulation for children with cerebral palsy, Part 2: Upper extremity. Phys Ther 73: 514-522.

7. Dodd KJ, Taylor NF, Damiano DL (2002) A systematic review of the effectiveness of strength-training programs for people with cerebral palsy. Arch Phys Med Rehabil 83: 1157-1164.

8. Andersson C, Grooten W, Hellsten M, Kaping K, Mattsson E (2003) Adults with cerebral palsy: walking ability after progressive strength training. Dev Med Child Neurol 45: 220-228.

9. Fowler EG, Ho TW, Nwigwe AI, Dorey FJ (2001) The effect of quadriceps femoris muscle strengthening exercises on spasticity in children with cerebral palsy. Phys Ther 81:1215-1223.

10. Van der Linden ML, Hazlewood ME, Aitchison AM, Hillman SJ, Robb JE (2003) Electrical stimulation of gluteus maximus in children with cerebral palsy: Effects on gait characteristics and muscle strength. Dev Med Child Neurol 45: 385-390.

11. Daichman J, Johnston TE, Evans K, Tecklin JS (2003) The effects of a neuromuscular electrical stimulation home program on impairments and functional skills of a child with spastic diplegic cerebral palsy: A case report. Pediatr Phys Ther 15: 153-158.

12. Arora L (2014) Effect of electrical stimulation on spasticity in spastic diplegic cerebral palsy children international journal of innovative research and development. 395-400. 